Removable silicon insertion stiffeners for neural probes using polyethylene glycol as a biodissolvable adhesive

S. H. Felix, K. G. Shah, D. M. George, V. M. Tolosa, A. C. Tooker, H. J. Sheth, T. L. Delima, S. S. Pannu

March 29, 2012

International Conference of the IEEE Engineering in Medicine and Biology Society

San Diego, CA, United States

August 28, 2012 through September 1, 2012 
This document was prepared as an account of work sponsored by an agency of the United States government. Neither the United States government nor Lawrence Livermore National Security, LLC, nor any of their employees makes any warranty, expressed or implied, or assumes any legal liability or responsibility for the accuracy, completeness, or usefulness of any information, apparatus, product, or process disclosed, or represents that its use would not infringe privately owned rights. Reference herein to any specific commercial product, process, or service by trade name, trademark, manufacturer, or otherwise does not necessarily constitute or imply its endorsement, recommendation, or favoring by the United States government or Lawrence Livermore National Security, LLC. The views and opinions of authors expressed herein do not necessarily state or reflect those of the United States government or Lawrence Livermore National Security, LLC, and shall not be used for advertising or product endorsement purposes. 


\title{
Removable Silicon Insertion Stiffeners for Neural Probes Using Polyethylene Glycol As a Biodissolvable Adhesive
}

\author{
Sarah Felix, Member, IEEE, Kedar Shah, Diana George, Vanessa Tolosa, Angela Tooker, Heeral \\ Sheth, Terri Delima, Satinderpall Pannu
}

\begin{abstract}
Flexible polymer probes are expected to enable extended interaction with neural tissue by minimizing damage from micromotion and reducing inflammatory tissue response. However, their flexibility prevents them from being easily inserted into the tissue. This paper describes an approach for temporarily attaching a silicon stiffener with biodissolvable polyethylene glycol (PEG) so that the stiffener can be released from the probe and extracted shortly after probe placement. A novel stiffener design with wicking channels, along with flipchip technology, enable accurate alignment of the probe to the stiffener, as well as uniform distribution of the PEG adhesive. Insertion, extraction, and electrode function were tested in both agarose gel and a rat brain. Several geometric and material parameters were tested to minimize probe displacement during stiffener extraction. We demonstrated average probe displacement of $28 \pm 9 \mu \mathrm{m}$.
\end{abstract}

\section{INTRODUCTION}

Microelectrode neural interfaces are an essential tool in neuroscience as well as emerging clinical applications such as prosthetics. In particular, penetrating micro-electrode probes enable stimulation and recording of neuronal activity in the brain and spinal cord. While conventional microelectrode probes made of material such as silicon or tungsten are adequate for acute studies, stimulation performance and recording signals typically degrade over time [1]. Modeling and experimental studies of the interaction between microelectrode probes and neural tissue reveal several mechanisms for this degradation, including micro-tearing and immune response [2-4]. It has been widely concluded that flexible probes that match more closely the bulk stiffness properties of neural tissue can minimize relative micromotion that can cause damage [3]. In addition, probes with a smaller overall size reduce the extent of tissue inflammation [4]. To achieve these characteristics of flexibility and minimal size, biocompatible polymers such as polyimide and parylene have been adopted as an ideal substrate for microelectrode probes [5-7].

A major challenge with flexible polymer probes is that it is difficult to insert them into the neural tissue. Flexible microscale probes tend to buckle upon insertion [8].

*Research supported by National Institute of Deafness and Other Communication Diseases.

S. Felix is with Lawrence Livermore National Laboratory, Livermore, CA 94550 USA (phone: 925-423-4921; fax: 925-422-2373; e-mail: felix5@1lnl.gov).

K. Shah, D. George. V. Tolosa, A. Tooker, H. Sheth, T. Delima, and S Pannu are with Lawrence Livermore National Laboratory, Center for Micro- and Nano-Technology, Livermore, CA 94550 USA (e-mail: shah22@1lnl.gov, george27@1lnl.gov, tolosa1@1lnl.gov, tooker1@1lnl.gov, sheth2@1lnl.gov, delima1@1lnl.gov, pannu1@1lnl.gov).
Researchers have attempted to develop methods to facilitate insertion of flexible probes. Early studies reporting results with polymer probes achieved implantation by permanently integrating the probe with a stiff material such as silicon or tungsten [9]. However, this negates the flexibility originally intended for the polymer probe. More complicated designs modify the polymer probe geometry to incorporate ribs or layers of other materials such that the device has increased stiffness in certain sections or axes, while maintaining compliance in other parts $[10,11]$. Yet another approach integrates a 3-D channel into the polymer probe design that is filled with biodegradable material [12]. This probe can be temporarily stiffened, and after insertion the material in the channel dissolves and drains out. All of the approaches just mentioned permanently modify the geometry of the final implanted device, compromising the desirable features of the flexible planar probe.

Methods that do not alter the geometry or function of the probe itself are more advantageous. One method to accomplish this is to encapsulate the polymer device with biodegradable material to temporarily stiffen the device $[8,13]$. However, typical biodegradable materials have Young's moduli orders of magnitude smaller than that of silicon and would consequently require larger dimensions to achieve the same stiffness. Also, coating the probe leads to rounding of the tip, making insertion more difficult. One promising method reported is to coat a stiffening shuttle with a self-assembling monolayer (SAM) to customize the surface interaction between the shuttle and the flexible probe [14]. When dry, the probe adheres to the coated shuttle electrostatically. After insertion, water migrates onto the hydrophilic surface, separating the probe from the shuttle so that the shuttle can be extracted. While shuttle extraction with reduced probe displacement was demonstrated $(85 \mu \mathrm{m})$, this method has several limitations. With only electrostatic interactions holding the probe to the shuttle, there is a risk of probe slippage relative to the shuttle during insertion. For the same reason, it is not possible to have any part of the polymer probe overhanging the stiffener. This means that alignment is critical unless the stiffener is oversized to contain the footprint of the probe while tolerating the significant misalignment that results from manual assembly. Moreover, this limitation eliminates designs that could minimize the cross-section of the stiffener by making its footprint smaller than that of the probe.

We have developed a method in which the flexible probe is attached to a stiffener with a temporary biodissolvable adhesive material that securely holds the probe during insertion. Once inserted into the tissue, the 
adhesive material dissolves and the stiffener is extracted to restore the probe to its intended flexibility. The probe and stiffener geometry are fully decoupled such that the stiffener footprint does not have to match or be larger than that of the probe. This advantageous approach is enabled by a novel stiffener design and methodical assembly process which ensure uniform adhesive coverage, accurate and repeatable alignment, and no overflow of adhesive to the functional side of the probe.

We selected polyethylene glycol (PEG) as the biodissolvable adhesive material. PEG has been used in implanted applications such as neural probes and drug delivery $[12,15]$. Compared to other possible materials, including sucrose and PLGA, PEG has a dissolution time in biological fluids that is of an appropriate scale for implant surgeries (on the order of tens of minutes, depending on molecular weight). In addition, it is solid at room temperature and liquid at temperatures ranging from 50-65 ${ }^{\circ} \mathrm{C}$. This behavior makes it particularly suitable for our precision assembly process.

Using PEG to adhere a flexible neural probe to a silicon stiffener has been mentioned briefly in literature, but with limited results reported [6]. This may be because attempted methods have been largely manual, which makes attachment of microscale devices with adhesive unreliable. We have demonstrated that with novel stiffener design, well-tuned assembly procedures, and adhesive material optimization, this approach is a viable option to temporarily stiffen polymer probes for insertion. Success of this approach was demonstrated by evaluating electrode function, insertion, and stiffener extraction in an agarose gel model of neural tissue, as well as a preliminary in vivo test in a rat.

\section{METHODS}

\section{A. Stiffener design and assembly process}

The silicon stiffener design is illustrated in Fig. 1. A large tab allows for handling the stiffener without contacting the attached probe. Channels are etched into the top surface of the stiffener, connected to a reservoir on the tab. These channels exploit capillary forces in order to distribute precise amounts of adhesive along the length of the stiffener prior to attachment of the neural probe. The stiffeners were fabricated using standard microfabrication processing. The assembly process flow is illustrated in Fig. 2. First, micro-

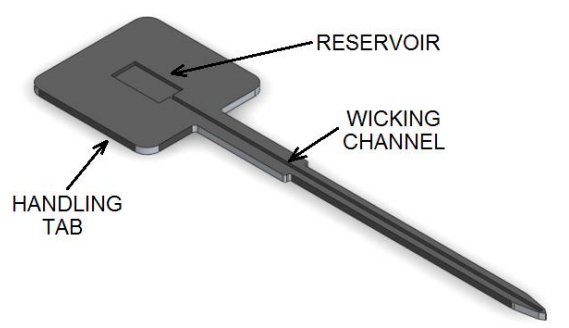

Figure 1. Silicon stiffener with large handling tab containing a reservoir well, and narrow shank with a channel to wick liquid adhesive. pellets of PEG are generated by melting flakes of the material on a hydrophobic surface. Then the PEG is drawn and spread to create separate droplets of a desired size, and then allowed to solidify. Pellets are picked and placed into the reservoir on the tab portion of the stiffener (Fig. 2a). The stiffener is then placed on a hot plate, where the PEG melts and wicks into the channel (Fig. 2b). When wicking is completed, the stiffener is cooled and the PEG solidifies. The stiffener can then be easily handled and aligned to the probe using a flip-chip bonder without the risk of wet adhesive transferring to other surfaces in the process (Fig. $2 \mathrm{c})$. Once the probe and stiffener are aligned and placed into contact, the assembly is heated again causing the PEG to wick out of the channels and into the interface between the probe surface and the top surface of the stiffener. Finally, the device is cooled, re-solidifying the PEG, to complete the assembly (Fig. 2d).

Fig. 3 shows a flexible probe aligned and attached to a silicon stiffener with PEG. The probe and stiffener are aligned to within $10 \mu \mathrm{m}$, based on calibration of the flip-chip tool. PEG has uniformly filled the entire area under the polymer probe, and no PEG has migrated to the top surface of the probe.

\section{B. Insertion/extraction testing in agarose gel}

As a neural tissue model, $0.6 \%$ agarose gel was prepared in a solution of phosphate buffered saline (PBS) and incubated at $37^{\circ} \mathrm{C}$. This agar concentration models the consistency of brain tissue $[12,16]$, while the PBS provides the salinity and $\mathrm{pH}$ of the bodily fluid. Polymer probes attached to silicon stiffeners were inserted into the tissue model using a manually-operated stereotaxic frame. Fig. 4 shows a schematic of the insertion and extraction process. First, the stiffened probe is attached to a stereotaxic frame and lowered into the model tissue (Fig. 4A). Typically, the top of

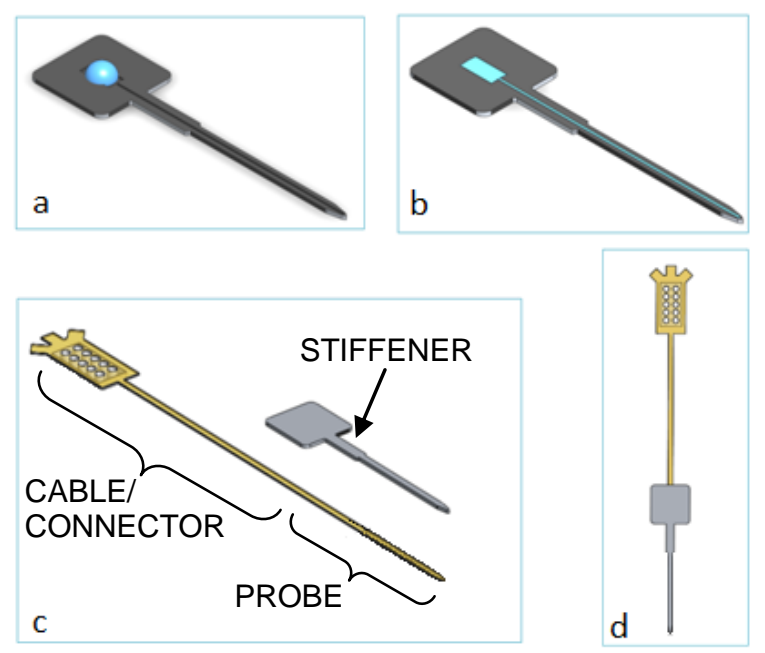

Figure 2. Process to assemble a flexible polymer probe to a silicon stiffener with polyethylene glycol. A) Pellet of solid PEG is placed into the reservoir on the tab of the stiffener. B) When heated, PEG melts and wicks into the channel. C) After resolidification, the stiffener can be aligned and mated to the probe using a flip-chip bonder. D) Completed assembly. 


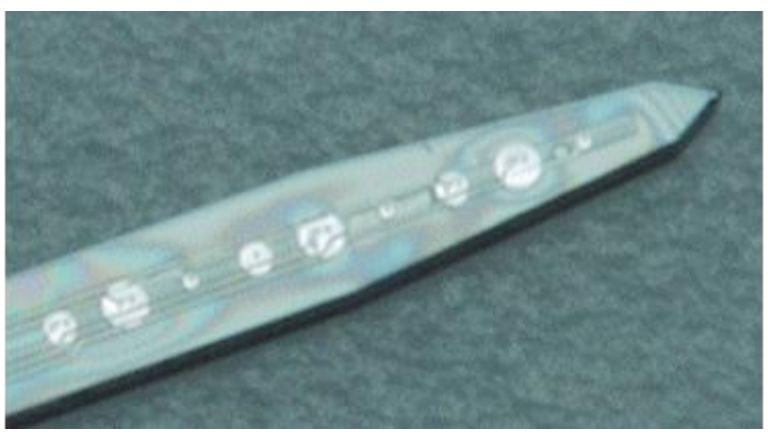

Figure 3. Flexible neural probe aligned and attached to a silicon stiffener with a wicking channel. PEG adhesive completely covers the underside of the probe without migrating to the top surface of the probe.

the flexible probe includes an electronic connector that is also secured to the stereotaxic frame. The connector end of the polyimide probe is moved from the stereotaxic frame to dissolving the adhesive (Fig. 4C). After the adhesive is dissolved, the stiffener is extracted from the tissue using the stereotaxic frame (Fig. 4D). This experiment was repeated using different PEG molecular weights (6000, 10000, and $20000 \mathrm{~g} / \mathrm{mol}$ ). Time allowed for the PEG to dissolve was varied from five minutes to 30 minutes. In addition, several different stiffener thicknesses $(20,50$ and $100 \mu \mathrm{m})$, widths, wicking channel configurations (1- and 3- channel), and extraction speeds were tested. Combinations of these parameters were sampled and varied adaptively until a suitable combination was identified. Then, the favorable set of parameters was tested five times to evaluate repeatability. During the insertion and extraction in agarose gel, the probe assembly was observed using a Hirox microscope and camera. A clear glass chip with a grid of dots was placed into the gel about a millimeter in front of the probe insertion site. This grid served as a fiducial marker to compare images of the probe before and after extraction of the stiffener, and thereby measure probe displacement. Average resolution was about 2 micrometers per pixel.

\section{In vivo neural recording test}

We used the proposed removable insertion method to implant a polyimide-based probe with $20-\mu \mathrm{m}$ diameter platinum electrodes into the prefrontal cortex of an anesthetized rat. The probe was attached with PEG of molecular weight $10,000 \mathrm{~g} / \mathrm{mol}$, and then sterilized in ethylene oxide at room temperature. After removing the dura mater, the probe-stiffener assembly was guided $4 \mathrm{~mm}$ into the brain tissue with a manually driven stereotaxic frame. After 15 minutes, the stiffener was slowly extracted. After a day of post-surgery recovery time, neuronal activity was recorded.

\section{Electrochemical testing}

The flexible polymer devices in this study included 16channel activated iridium oxide electrodes. Electrochemical testing was performed to ensure that the assembly process and subsequent insertion/extraction did not degrade the performance of these electrodes.

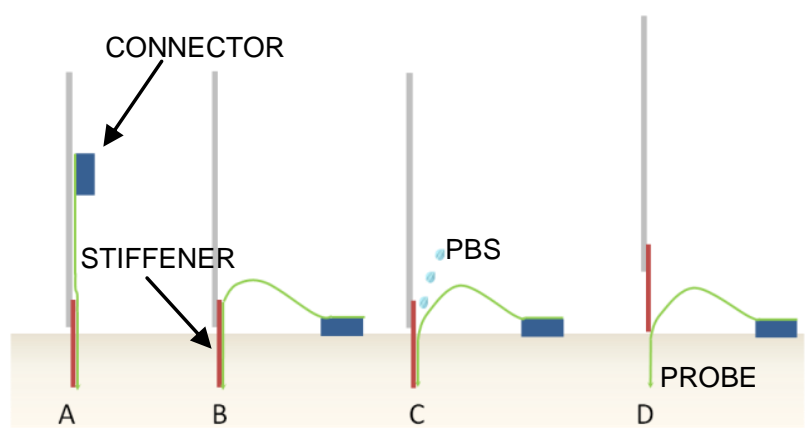

Figure 4. Process for inserting the stiffened probe and subsequently extracting the stiffener. The arm of a stereotaxic frame supports the probe assembly as it is lowered into the tissue. PBS is applied to help dissolve the adhesive so that the stiffener can be extracted, leaving the probe in place.

Common parameters measured for neural stimulating electrodes are the charge storage capacity (CSC) and impedance. Cyclic voltammetry (CV) was performed between $-600 \mathrm{mV}$ and $+800 \mathrm{mV}$ at a scan rate of $100 \mathrm{mV} / \mathrm{s}$. CSC was calculated from the time integral of the cathodic current during the potential sweep. Electrochemical impedance spectroscopy (EIS) data was measured from $1 \mathrm{~Hz}$ to $3 \mathrm{MHz}$ using a $10 \mathrm{mV}$ rms sine wave. CV and EIS measurements were made with a Princeton Applied Research (PAR) potentiostat using vendor-supplied software.

CSC and EIS were measured at three time points. Initial measurements were made after activation in PBS. After assembly, the stiffened probes were inserted into the agarose gel prepared with PBS. CSC and EIS were again measured immediately after insertion. Then the PEG was allowed to dissolve approximately 15 minutes and the stiffener was carefully extracted by hand. Measurements were repeated after stiffener extraction.

\section{RESULTS AND DISCUSSION}

Results from electrochemical testing indicate that the electrode performance is not degraded following the assembly process, insertion, and stiffener extraction. Table 1 lists the CSC and impedance values at different stages.

Initial testing revealed that for a given PEG molecular weight, the time allowed to dissolve strongly influenced how easily the stiffener detached from the probe. The influence of stiffener thickness, width, and channel configuration were not immediately clear. Finally, we observed that varying extraction speed helped the stiffener to separate from the probe, reducing probe displacement. A favorable set of parameters included PEG with molecular weight 10,000 $\mathrm{g} / \mathrm{mol}$, a dissolution time of 15 minutes, and the optimized extraction speed profile. These parameters were tested five times, with an average probe relative displacement of $28 \pm 9$ $\mu \mathrm{m}$. Fig. 5 shows a representative set of images comparing probe location before and after stiffener extraction.

During the in vivo test in an anesthetized rat, a small amount of probe displacement was observed visually during extraction of the stiffener. This was estimated to be roughly 100 microns, although it could not be measured precisely. Note that this test did not apply the optimized extraction speed profile used in the agarose gel tests. 
TABLE 1: ELECTROCHEMICAL MEASUREMENTS

\begin{tabular}{|l|c|c|}
\hline & $\begin{array}{c}\mathrm{CSC} \\
{\left[\mathrm{mC} / \mathrm{cm}^{2}\right]}\end{array}$ & $\begin{array}{c}\text { Impedence at 1 } \\
\mathrm{kHz} \\
{[\mathrm{kOhm}]}\end{array}$ \\
\hline $\begin{array}{l}\text { After initial iridium oxide } \\
\text { activation in PBS }\end{array}$ & $23.84 \pm 0.78$ & $4.53 \pm 0.25$ \\
\hline $\begin{array}{l}\text { After insertion into agarose } \\
\text { gel with stiffener attached }\end{array}$ & $25.92 \pm 0.89$ & $5.34 \pm 0.20$ \\
\hline After extraction of stiffener & $24.93 \pm 0.89$ & $5.11 \pm 0.20$ \\
\hline
\end{tabular}

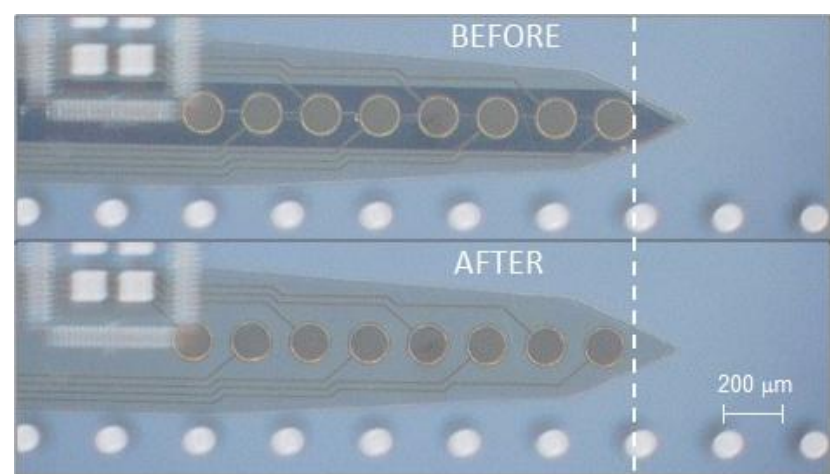

Figure 5. Images of the probe before (top) and after (bottom) stiffener extraction, positioned side by side for clarity. The dark strip in the top image is the stiffener. This probe moved 35 microns to the left.

Nonetheless, after recovery, the flexible probe successfully recorded single neuron spikes on several channels as well as local field potential on the majority of channels. The neural activity from one of these channels is shown in Fig. 6.

\section{CONCLUSION}

We have demonstrated the viability of using polyethylene glycol as a biodissolvable adhesive for temporarily attaching flexible neural probes to insertion stiffeners. Our novel stiffeners designed with wicking channels, along with flip chip assembly, ensured uniform bond coverage with accurate and repeatable alignment between the stiffener and the probe. We demonstrated extraction of the stiffener with average probe displacement of $28 \pm 9 \mu \mathrm{m}$ in an agarose gel model. Promising preliminary in vivo results indicate that this method could enable truly flexible implanted probes. Further testing of the various parameters is in progress to optimize the proposed method. In addition, further in vivo testing is necessary for both acute evaluation of electrode placement and function, as well as long term studies to quantify the benefits of removable stiffeners

\section{ACKNOWLEDGMENT}

The authors thank Loren Frank at UCSF for facilitating our in vivo trial and providing the neural recording data. This work was performed under the auspices of the U.S. Department of Energy by Lawrence Livermore National Laboratory under Contract DE-AC52-07NA27344. LLNLCONF-542891.

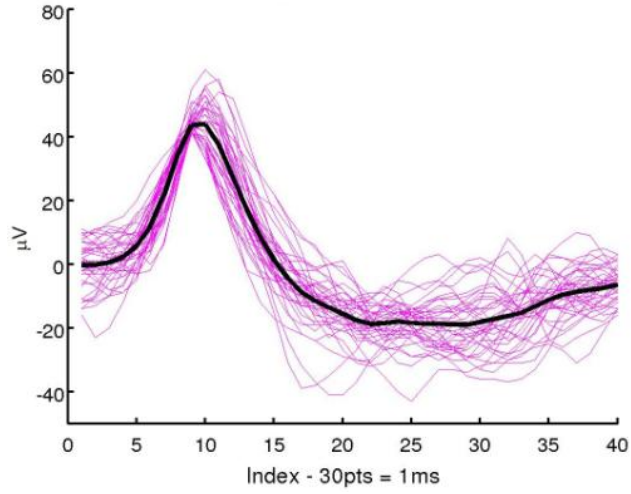

Figure 6. Electrodes were able to record single neuron spikes after the PEG adhesive was allowed to dissolve and the stiffener was removed.

\section{REFERENCES}

[1] X. Liu, D. B. McCreery, L. A. Bullara, and W. F. Agnew, "Evaluation of the stability of intracortical microelectrode arrays," IEEE Trans. Neural Sys. and Rehab. Engineering, vol. 14, 2006, pp. 91-100.

[2] R. Biran, D. Martin, and P. Tresco, "Neuronal cell loss accompanies the brain tissue response to chronically implanted silicon microelectrode arrays," Exp. Neurology, vol. 195, pp. 115-126, 2005.

[3] V. Polikov, P. Tresco, and W. Reichert, "Response of brain tissue to chronically implanted neural electrodes," J. Neurosci. Meth., vol. 148, 2005, pp. 1-18.

[4] D. H. Szarowski et al., "Brain responses to micro-machined silicon devices," Brain Research, vol. 983, 2003, pp. 23-35.

[5] Y. Sun, S. Lacour, R. Brooks, R. Rushton, J. Fawcett, and R. Cameron, "Assessment of the biocompatibility of photosensitive polyimide for implantable medical device use," J. Biomed. Mat. Res. $A$, vol. 90, no. 3, September 2009, pp. 648-655.

[6] D. R. Kipke, D. S. Pellinen, R. J. Vetter, "Advanced neural implants using thin-film polymers," IEEE Int. Symp. On Circuits and Systems, vol. 4, 2002, pp. 173-176.

[7] A. Mercanzini, K. Cheung, D. Buhl, M. Boers, A. Maillard, P. Colin, J.-C. Bensadoun, A. Bertsch, and P. Renaud, "Demonstration of cortical recording using novel flexible polymer neural probes," Sens. Actuators A, vol. 143, 2008, pp. 90-96,

[8] A. Singh, H. Zhu, J. He, "Improving mechanical stiffness of coated benzocyclobutene (BCB) based neural implant," Int' Conf. Engineering in Medicine and Biology Society, Sept. 2004, pp. 42984301.

[9] D. P. O'Brian, T. R. Nichols and M. G. Allen, "'Flexible microelectrode arrays with integrated insertion devices, " $14^{\text {th }}$ IEEE Int'l. Conf. on Microelectromechanical Systems, Technical Digest, 2001, pp. 216-219.

[10] D. Egert, R. L. Peterson and K. Najafi, "Parylene microprobes with engineered stiffness and shape for improved insertion," Transducers '11, Beijing, China, June 5-9, 2011, pp. 198-201.

[11] K.-K. Lee, et al, "Polyimide-based intracortical neural implant with improved structural stiffness," J. Micromechanics and Microengineering, vol. 14, 2004, pp. 32-37.

[12] S. Takeuchi, D. Ziegler, Y. Yoshida, K. Mabuchi, and T. Suzuki, "Parylene flexible neural probes integrated with microfluidic channels," Lab On A Chip, vol. 5, 2005, pp. 519-523.

[13] D. Lewitus, K. L. Smith, W. Shain, J. Kohn, "Ultrafast resorbing polymers for use as carriers for cortical neural probes," Acta Biomaterialia, vol. 7, 2011, pp. 2483-2491.

[14] T. D. Y. Kozai and D. R. Kipke, "Insertion shuttle with carboxyl terminated self-assembled monolayer coatings for implanting flexible polymer neural probes in the brain, "J. Neuroscience Methods, 2009.

[15] R. B. Greenwalk, Y. H. Choe, J. McGuire and C. D. Conover, "Effective drug delivery by PEGylated drug conjugates," Advanced Drug Delivery Reviews, vol. 65, no. 2, Feb. 2003, pp. 217-250.

[16] Z.-J. Chen et al., "A realistic brain tissue phantom for intraparenchymal infusion studies," J. Neurosurg, vol. 101, 2004, pp. 314-322. 